\title{
THE INTERMEDIATE PRIME DIVISORS OF INTEGERS
}

\author{
J.-M. DE KONINCK AND J. GALAMBOS
}

\begin{abstract}
Let $p_{1}<p_{2}<\cdots<p_{\omega}$ be the distinct prime divisors of the integer $n$. If $\omega=\omega(n) \rightarrow+\infty$ with $n$, then $p_{\text {, }}$ is called an intermediate prime divisor of $n$ if both $j$ and $\omega-j$ tend to infinity with $n$. We show that $\log \log p_{j}$, as $j$ goes through the indices for which $p_{j}$ is intermediate, forms a limiting Poisson process in the sense of natural density.
\end{abstract}

Let $p_{1}<p_{2}<\cdots<p_{\omega}$ be the distinct prime divisors of the integer $n$. Here, of course, $p_{j}=p_{j}(n)$ and $\omega=\omega(n)$. Our interest is to establish certain properties of the prime divisors $p_{j}$ which are valid with positive density. Recall that density is defined as follows. Let $\nu_{N}(\cdots)$ be the number of integers $1 \leqslant n \leqslant N$ for which the property stated in the dotted space holds. Set $P_{N}(\cdots)=\nu_{N}(\cdots) / N$. Then the density $d(A)$ of $A$ is defined as

$$
d(A)=\lim P_{N}(A) \quad(N \rightarrow+\infty) .
$$

In particular, the simplest results of probabilistic number theory (see Elliott [2, Introduction]) imply that, with density one, $\omega(n) / \log \log n$ is asymptotically one. Hence, with density one, we can distinguish three types of prime divisors: we call $p_{j}$ small if $j$ is bounded as $n \rightarrow+\infty, p_{j}$ large if $\omega-j$ remains bounded, and all others intermediate. For the investigation of the small prime divisors, tools of elementary number theory suffice. Large prime divisors require special tools, but very old results (due to Dickman, see De Koninck and Ivic [1] for accurate statements and for asymptotic formulas involving large prime divisors) tell us that $\left(\log p_{j}\right) / \log n$ falls into the interval $(a, b), 0 \leqslant a<b \leqslant 1$, with positive density for $j=\omega$. Extensions are also known for all large prime divisors, and the results are similar in nature. This perhaps explains why it was 'necessary' and so successful in probabilistic number theory to truncate additive functions at $r=r(N)$ with $(\log r) / \log N \rightarrow 0$ : it simply cancels the effect of the large prime divisors (see Elliott [3], particularly Chapter 12). It indeed required a completely new method of attack when the truncation was abandoned and new types of results were obtained (once again, see [3]). The truncation methods, in which the intermediate prime divisors contributed all the influence for the validity of a statement, already show that the intermediate prime divisors behave asymptotically as independent random variables. The fact that this asymptotic independence is even stronger than what follows from

Received by the editors July $1,1986$.

1980 Mathematics Subject Classification (1985 Revision). Primary 10K20: Secondary 10H15. 
additive functions is the subject of the present paper. It is related to an earlier result of Galambos [4], stating that, for intermediate terms, $\log \log p_{j+1}(n)-\log \log p_{j}(n)$ are asymptotically unit exponential variables, that is, the density for which the just stated difference is smaller than a positive value $z$ equals $1-e^{-z}$. The remarkable part of this result is that the density does not depend on $j$. Maier [7] extended this result to showing that a finite set of the above differences are asymptotically independent in the sense of probability theory. We further generalize these results by proving the following statement.

THEOREM 1. Let $j=j(N)$ be a positive integer valued function tending to infinity with $N$. Assume that $j$ is such that, with perhaps the exception of a set of density zero, $p_{j}(n) \rightarrow+\infty$ with $N$, and $\left\{\log p_{j}(n)\right\} / \log N \rightarrow 0$ as $N \rightarrow+\infty$, where $1 \leqslant n \leqslant N$. Then the points $\log \log p_{j+k}, k \geqslant 1$, form a Poisson process in limit as $N \rightarrow+\infty$.

Before proceeding to the proof, let us comment on the restrictions on $j$. These are exactly for making sure that we deal with intermediate terms. As a matter of fact, $p_{j} \rightarrow+\infty$ excludes the small prime divisors, and, in view of Dickman's result, $\left\{\log p_{j}(n)\right\} / \log N \rightarrow 0$ excludes the large ones.

The proof relies on the following result which is of interest on its own.

THEOREM 2. Let $T_{N}$ be a set of primes $q$ such that if $q \in T_{N}$ then $q \rightarrow+\infty$ with $N$, and for every fixed $k, q^{k} \leqslant N$. Furthermore, we assume that there is a number $0<\lambda<+\infty$ such that

$$
\sum_{q \in T_{N}} \frac{1}{q} \rightarrow \lambda \text { as } N \rightarrow+\infty .
$$

Then, if $m_{N}(n)$ denotes the number of (distinct) prime divisors of $n$ from the set $T_{N}$, we have for every $r=0,1,2, \ldots$, as $N \rightarrow+\infty$,

$$
\lim P_{N}\left(m_{N}(n)=r\right)=\lambda^{r} e^{-\lambda} / r ! \text {. }
$$

Proof of Theorem 2. There is an elementary result in probability theory (see, for example, $\$ 5.5$ in [5]) which states that for the validity of (2) it suffices to show that, for every fixed $k, S_{k, N}\left(T_{N}\right) \rightarrow \lambda^{k} / k !$ as $N \rightarrow+\infty$, where

$$
S_{k, N}\left(T_{N}\right)=\sum P_{N}\left(e_{i_{1}}(n)=e_{i_{2}}(n)=\cdots=e_{i_{k}}(n)=1\right),
$$

the summation being extended over all $i_{1}<i_{2}<\cdots<i_{k}$ with $q_{i} \in T_{N}$ and

$$
e_{j}(n)= \begin{cases}1 & \text { if } q_{j} \mid n, \\ 0 & \text { otherwise. }\end{cases}
$$

Now, clearly, the general term on the right-hand side of (3) equals

$$
\frac{1}{N}\left[\frac{N}{q_{i_{1}} q_{i_{2}} \ldots q_{i_{h}}}\right] \text {, }
$$

where $[y]$ signifies the integer part of $y$. By our assumptions on $T_{N}$, each expression above is positive, and the number of terms in the sum at (3) is $o(N)$. Thus

$$
S_{k, N}\left(T_{N}\right)=\sum \frac{1}{q_{i_{1}} q_{i_{2}} \ldots q_{i_{k}}}+o(1), \quad k \geqslant 1,
$$


where $\sum$ is as in (3). Hence, (1) and (4) yield, as $N \rightarrow+\infty$,

$$
\lim S_{1, N}\left(T_{N}\right)=\lambda \text {. }
$$

Next, observe that, for $k \geqslant 2$,

(6) $S_{1, N}\left(T_{N}\right) S_{k-1, N}\left(T_{N}\right)=k S_{k, N}\left(T_{N}\right)+O\left(Q_{T} S_{k-1, N}\left(T_{N}\right)\right)+o\left(S_{k-1, N}\left(T_{N}\right)\right)$,

where $Q_{T}=1 / q(T)$ with $q(T)$ being the smallest member of $T_{N}$. By assumption, $Q_{T} \rightarrow 0$ as $N \rightarrow+\infty$. Thus, by induction, (5) and (6) yield the desired limit for $S_{k, N}\left(T_{N}\right)$. The proof is complete.

ProOF OF THEOREM 1. Let us choose an arbitrary $j=j(N)$ satisfying the conditions of the theorem. We fix this function, and $\log \log p_{j}(n)$ serves as the starting point of the point process, to be shown to be Poisson in limit. Let $\left(t_{m, N}, t_{m, N}^{*}\right), 1 \leqslant m \leqslant M, M$ fixed, be a finite number of disjoint intervals such that, as $N \rightarrow+\infty$, both $t_{m, N}$ and $t_{m, N}^{*}$ tend to some finite points $t_{m}$ and $t_{m}^{*}$, respectively, and the intervals $\left(t_{m}, t_{m}^{*}\right), 1 \leqslant m \leqslant M$, remain disjoint. We form the intervals

$$
\left(\log \log p_{j}(n)+t_{m, N}, \log \log p_{j}(n)+t_{m, N}^{*}\right), \quad 1 \leqslant m \leqslant M,
$$

and count the number $m_{N}\left(M ; t, t^{*}\right)$ of $k$ such that $\log \log p_{j+k}(n)$ falls into one of the intervals at (7). By the result of Rényi [8] it suffices to prove that the asymptotic distribution of $m_{N}\left(M ; t, t^{*}\right)$ is Poisson whose $\lambda$-parameter is the sum of the $\lambda$-parameters of the asymptotic (Poisson) distribution of the number of $k$ 's for the individual intervals at (7). However, this limiting result follows from Theorem 2 upon observing that the inequalities

$$
\log \log p_{j}(n)+t_{m, N} \leqslant \log \log p_{j+k}(n) \leqslant \log \log p_{j}(n)+t_{m, N}^{*}
$$

are equivalent to

$$
\left(p_{j}(n)\right)^{\gamma_{m, v}} \leqslant p_{j+k}(n) \leqslant\left(p_{j}(n)\right)^{\gamma_{m, N}^{*}},
$$

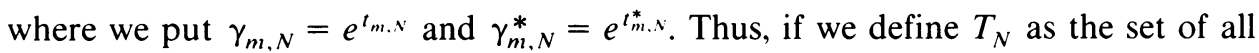
primes $q$ such that $\log \log q$ falls into one of the intervals at (7), $m_{N}\left(M ; t, t^{*}\right)$ becomes $m_{N}(n)$ of Theorem 2. Condition (1), as well as the required additivity of $\lambda$ over the intervals of (7), follows from the elementary asymptotic formula (see Hardy and Wright [6])

$$
\sum_{q \leqslant N} \frac{1}{q}=\log \log N+C+o(1)
$$

in view of the relation of (7) to (8). All other conditions of Theorem 2 are satisfied by assumption, and, therefore, the theorem is established.

\section{REFERENCES}

1. J.-M. De Koninck and A. Ivic, Topics in arithmetical functions, Notas de Matematica 72, NorthHolland, Amsterdam, 1980.

2. P. D. T. A. Elliott, Probabilistic number theory. I, Springer-Verlag, Berlin and New York, 1979.

3. Probabilistic number theory. II, Springer-Verlag, Berlin and New York, 1980. 
4. J. Galambos, The sequences of prime divisors of integers, Acta Arith. 31 (1976), 213-218.

5. _ Introductory probability theory, Marcel Dekker, New York, 1984.

6. G. H. Hardy and E. M. Wright, An introduction to the theory of numbers, 3rd ed., Oxford Univ. Press, 1960.

7. H. Maier, On the set of divisors of an integer, Technical Report, Univ. of Michigan, Ann Arbor, 1983.

8. A. Rényi, Remarks on the Poisson process, Studia Sci. Math. Hungar. 2 (1967), 119-123.

Departement de Mathematique, Université Laval, Québec, Canada G1K 7P4

Department of Mathematics, Temple University, Philadelphia, Pennsylvania 19122 